\title{
Escala de percepção de esforço para criança (EPEC): validação para o português em um teste submáximo
}

http://dx.doi.org/10.11606/1807-5509202000030513

\author{
Renata MARTINS* \\ Renata Maba GONÇALVES* \\ Rafaela Coelho MINSKY* \\ Anamaria Fleig MAYER M/** $^{* *}$ \\ Camila Isabel Santos SCHIVINSKI ${ }^{* / * * *}$ \\ *Universidade do \\ Estado de Santa \\ Catarina, Florianópolis \\ SC, Brasil. \\ **Universidade Federal \\ de São Paulo, São \\ Paulo, SP, Brasil. \\ ***Universidade \\ Estadual de Campinas, \\ Campinas, SP, Brasil.
}

0 objetivo do presente estudo é traduzir e validar, para a língua portuguesa do Brasil, a escala de percepção de esforço para crianças (EPEC). Para isto, toda a etapa de tradução da PES-C, da língua inglesa para a portuguesa (do Brasil), foi realizada com base no protocolo proposto por Guillemin et al. ${ }^{19}$. Para validação da escala, esta foi aplicada durante a realização do teste de caminhada de seis minutos (TC6). A validade da EPEC foi avaliada com o uso do coeficiente de correlação de Pearson, para verificar o grau de associação com a escala de Borg. Para a estabilidade das respostas, calculou-se o erro padrão da média (EPM) e coeficiente de correlação intra-classe (ICC). Calculou-se o erro padrão da estimativa das medidas de percepção finais (EPE) de cada dia para analisar o grau de desvio dos dados obtidos. Para avaliação da fidelidade utilizouse 0 alpha de Cronbach, no sentido de se analisar a consistência interna deste instrumento. Neste estudo participaram do processo de validação da EPEC 36 crianças, com média de idade de $10 \pm 2,61$ anos, sendo $50 \%$ de meninas. No cálculo do EPM, encontrou-se um valor de 0,05 para o primeiro dia e 0,03 no segundo dia, caracterizando uma variabilidade baixa. 0 EPE foi de 0,33 no primeiro dia e 0,41 no segundo dia, demonstrando um erro baixo. A consistência interna apresentou coeficiente alfa de Cronbach de 0,865. A escala EPEC foi validada para a população de crianças brasileiras, demonstrando também ser reprodutivel em testes submáximos.

Palavras-chave: Reprodutibilidade; Jovem; Estresse; Tradução.

\section{Introdução}

A percepção do esforço resulta da integração de uma série de informaçóes aferentes oriundas de estruturas sensoriais localizadas nos músculos esqueléticos e no sistema cardiorrespiratório․ É considerada um indicador válido e confiável para monitorar e verificar a tolerância do indivíduo ao exercício ${ }^{2,3}$. A partir disso, escalas de percepção e esforço são criadas com o objetivo de estabelecer relaçóes entre a percepçáo subjetiva de esforço e os dados objetivos de carga externa, ou de estresse fisiológico ${ }^{3-5}$, bem como as escalas de dispneia que avaliam a sensação de dificuldade respiratória ${ }^{2,5}$.

Essas escalas são apresentadas de diversas formas: visuais analógicas, numéricas, de categorias verbais, com desenhos e híbridas ${ }^{5}$, sendo as mais utilizadas a Escala de Borg ${ }^{4}$, a Escala de Borg Modificada ${ }^{6}$ e a Escala de esforço percebido de Borg ${ }^{1}$. Os estudos referentes as escalas apresentam que, quanto mais componentes uma escala tiver, maior será a facilidade de entendimento durante sua aplicação ${ }^{2-6}$. No entanto, quando essas escalas são aplicadas em crianças, existe dificuldade na compreensão e na interpretação desses instrumentos, tornando o resultado referente à sensação de esforço não confiável. Apesar disso, assim como no adulto, normalmente se utiliza a escala modificada de Borg em crianças $^{7,8}$, apesar de a idade ser considerada um fator capaz de influenciar as respostas ao índice de esforço percebido ${ }^{9,10}$.

Nesse contexto, pesquisas têm utilizado escalas 
modificadas ${ }^{10-12}$ e novas escalas específicas estão sendo desenvolvidas para essa população, como é o caso da Escala visual analógica legendada de dispneia ${ }^{13}$, Children's Effort Rating Table - CERT (Tabela infantil de avaliação do esforço ${ }^{14}$, Pictorial Children's Effort Rating Table - PCERT (Tabela ilustrativa infantil de avaliação do esforço $)^{15}$, Children's OMNI Scale of Perceived Exertion (Escala OMNI de esforço percebido para crianças) ${ }^{16,17}$ e Perceived Exertion Scale for Children - PES-C (Escala de percepção de esforço para criança - EPEC) ${ }^{18}$. Todas essas escalas utilizam ilustrações, componente essencial na interpretação e

\section{Método}

\section{Sobre o instrumento}

A PES-C, é uma escala australiana desenvolvida em 2003 por Simon et al. ${ }^{18}$, validada para classificar a percepção de esforço em crianças de 6 à 7 anos de idade. Além de descritores verbais, possui seis ilustraçóes descritivas, posicionadas ao longo da escala, as quais se atribuem pontuaçóes que vão de zero (nem um pouco cansado) a cinco pontos (totalmente exausto, não posso continuar com o exercício). As ilustraçóes são representadas por um urso, cuja postura e expressáo escolha das crianças pelo item que melhor represente sua sensação de esforço ${ }^{18}$. Contudo, das cinco escalas citadas, apenas a primeira está disponível na língua portuguesa. Apesar de todas serem válidas para a população proposta, somente a PES-C apresentou valor de reprodutibilidade no artigo original de validação. Diante dessa escassez de instrumentos em português, o objetivo do presente estudo foi traduzir e validar a escala infantil australiana PES-C, para a língua portuguesa do Brasil (EPEC), a fim de atender crianças com idades entre 6 e 14 anos na avaliaçáo da sensação de esforço percebido.

facial se modificam a medida que a pontuação da escala aumenta, transmitindo a ideia de que o personagem desprende um esforço crescente para a atividade ou exercício $^{18}$ (FIGURA 1).

Para a validação do instrumento supracitado, utilizou-se a escala modificada de Borg, uma vez que esta escala abrange o mesmo constructo que a EPEC. A escala de Borg foi construída de forma vertical, quantificada de 0 a 10 , na qual 0 representa nenhuma sensação de dispneia/esforço e 10 representa o sintoma máximo de dispneia .

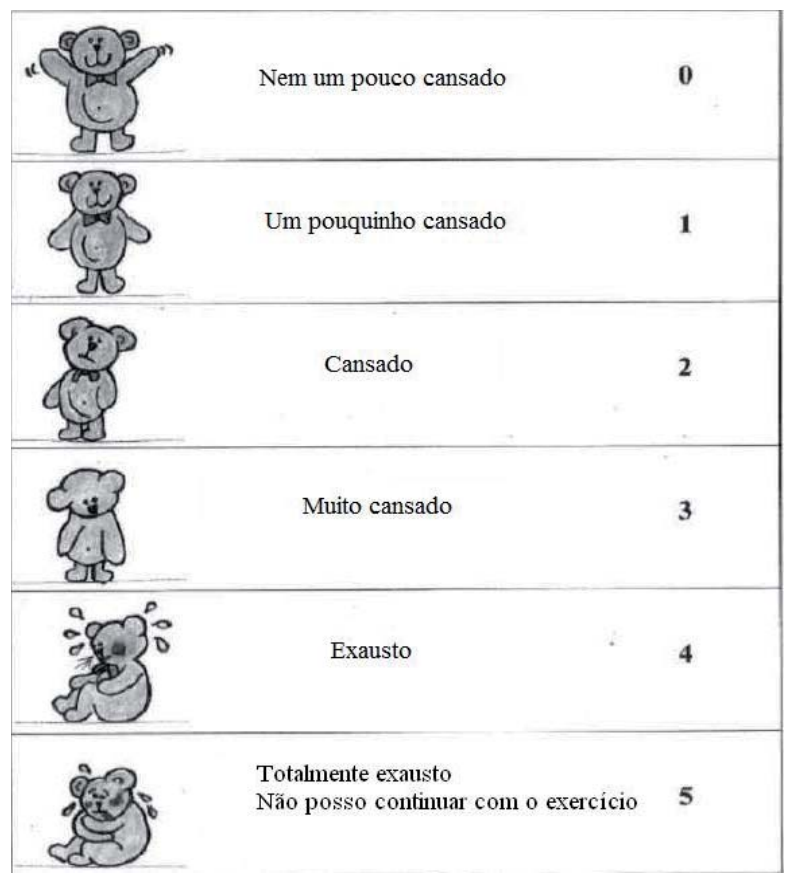

FIGURA 1 - Ilustrações da escala de percepção de esforço para crianças. 


\section{Tradução}

Toda a etapa de tradução da PES-C, da língua inglesa para a portuguesa (do Brasil), foi realizada com base no protocolo proposto por GUILLEMIN et al. ${ }^{19}$, o qual inclui a tradução inicial, a tradução reversa e a revisão por especialistas.

A tradução inicial da escala (em inglês) para a língua-alvo (português) foi feita por dois profissionais proficientes em ambos os idiomas, que não tiveram contato entre si. Os tradutores estavam cientes dos objetivos do estudo, sendo enfatizada, principalmente, a necessidade que fosse feita uma tradução conceitual, e não estritamente literária. Sendo assim, considerou-se o contexto no qual o uso original da escala estava inserido: de sua aplicação durante um teste de exercício submáximo.

Em seguida, as traduçôes foram comparadas, gerando uma única versão consensual denominada versão 1 , a qual foi submetida à traduçáo reversa ao inglês por dois tradutores fluentes, que desconheciam os objetivos do estudo e a versão original do instrumento. Essa nova versão (versão 2) foi comparada com o instrumento original e não apresentou discrepância.

$\mathrm{Na}$ etapa subsequente, dois especialistas da área da saúde revisaram a versão 1 da escala, para verificar equivalência semântica, idiomática e conceitual, considerando a utilidade da escala para avaliar a percepção de esforço durante um determinado teste de exercício. O objetivo foi identificar erros que levassem à diferença de significados, os quais foram reescritos até que houvesse um consenso. Garantiuse que todo texto apresentasse equivalência de sentido, sem comprometer a compreensão pela população a que se destinava. Assim foi gerada a versão final, utilizada para a validação.

\section{J ogos cooperativos}

Para validaçáo da escala, esta foi aplicada durante a realizaçáo do teste de caminhada de seis minutos (TC6), teste de exercício que despende esforço submáximo, de capacidade funcional, já consagrado na literatura ${ }^{20}$. Neste teste, frequentemente utilizado na população pediátrica, a avaliação da dispneia é padronizada, a qual comumente se dá através da Escala de Borg $^{4}$ e/ou Escala de Borg Modificada ${ }^{6}$. Para o corrente trabalho, utilizou-se também a PES-C ${ }^{18}$ traduzida (FIGURA 1), denominada entáo escala de percepção de esforço para crianças traduzida
(EPEC), cujo procedimento de validação seguiu a coleta de dados da pesquisa, descrita a seguir.

Após aprovação do Comitê de Ética da Universidade do Estado de Santa Catarina (UDESC) (CAAE: 07635412.3.0000.0118/ número do parecer interno: 102.162), a coleta de dados ocorreu nas dependências do Centro de Ciências da Saúde e do Esporte (CEFID) da UDESC e em escolas públicas e privadas da Grande Florianópolis, Santa Catarina - Brasil, nas quais as crianças estudavam, a fim de facilitar a aderência aos procedimentos de coleta de dados. Participaram da pesquisa crianças saudáveis com idades entre 6 e 14 anos. Para caracterização da higidez, as mesmas respeitaram os seguintes critérios: ausência de qualquer doença cardiorrespiratória, musculoesquelética, reumática, neurológica, e/ou déficits auditivos e visuais, não estarem inscritas em federações de esporte de alto rendimento (não atletas) ${ }^{21}$. Essas informaçóes foram obtidas por meio de um questionário recordatório de saúde elaborado pelas pesquisadoras e respondido pelos pais/responsáveis. Estes também responderam ao questionário International Study of Asthma and Allergies in Childhood (ISAAC) ${ }^{22}$, para identificação de asma (módulo 1) nas crianças. Houve exclusão dos participantes entre 6 e 9 anospara pontuaçóes $\geq 5$, e para $\geq 6$ pontos nas idades entre 10 e 14 anos. Aqueles que apresentaram alteração nos parâmetros espirométricos (volume expiratório forçado no primeiro segundo $\left[\mathrm{VEF}_{1}\right]$, capacidade vital forçada $[\mathrm{CVF}]<80 \%$ do previsto, e relação $\mathrm{VEF}_{1} / \mathrm{CVF}<0,7$, segundo a referência de Knudson et al. ${ }^{23}$ e Polgar et al. ${ }^{24}$, também foram excluídos da pesquisa.

Após a assinatura do termo de consentimento livre e esclarecido pelos pais/responsáveis, foi preenchida uma ficha de identificação. Subsequentemente, as crianças foram avaliadas quanto a dados biométricos (peso, estatura e índice de massa corporal ${ }^{25}$ ) e espirométricos ${ }^{26}\left(\mathrm{VEF}_{1}, \mathrm{CVF}\right.$ e relação $\mathrm{VEF}_{1} /$ CVF). Crianças que, por qualquer motivo, estiveram incapacitadas de executar ou concluir qualquer um dos procedimentos de avaliação e atividades propostas no dia da coleta de dados, não constituíram a amostra.

Os participantes foram então submetidos a dois TC6, seguindo as normas da American Thoracic Society ${ }^{20}$, com intervalo de 30 minutos entre eles. Para avaliação do índice de dispneia durante o teste foram utilizadas as escalas Modificada de Borg ${ }^{27}$ e a EPEC, esta segunda 
para validação. No segundo teste, as 2 escalas foram apresentadas e explicadas a cada criança por uma avaliadora diferente daquela que as aplicou no primeiro teste. A ordem de aplicação das escalas pelas avaliadoras foi randomizada. A randomização, não cega, aconteceu através de um dado (números pares representavam uma avaliadora e números ímpares à outra). Após um intervalo de $14 \mathrm{dias}^{28}$ foi realizado o teste-reteste e todo o procedimento de avaliação com o TC6 foi repetido.

\section{Análise dos dados}

Os dados foram analisados com o Programa SPSS para Windows, versão 20.0, e tratados com análise descritiva (média e desvio-padrão) e o sexo em frequências. O teste de Shapiro-Wilkfoi usado para verificar a normalidade dos dados. Aplicouse o coeficiente de correlação de Pearson entre o EPEC e a escala modificada de Borg para verificar o grau de associação entre as medidas.Para averiguar a estabilidade das respostas, calculouse o erro padrão da média (EPM), dado pela fórmula: $\mathrm{EPM}=\mathrm{DP} \times \sqrt{ }((1-I C C))$, onde $\mathrm{DP}$ é o desvio padrão e ICC é o coeficiente de correlação intraclasse. Calculou-se o erro padrão da estimativa das medidas de percepção finais (EPE)

\section{Resultados}

Um total de 58 crianças foi identificado para participar do estudo, sendo que dessas, 22 foram excluídas: 12 não realizaram a espirometria adequadamente ou tiveram os valores de $\mathrm{VEF}_{1}$ e/ou CVF menor que $80 \%$ do previsto, 2 apresentavam algum tipo de doença respiratória, 3 apresentaram pontuaçóes maiores que o ponto de corte no questionário ISAAC módulo de asma, 3 não quiseram finalizar os testes, 1 não tinha nacionalidade brasileira e 1 teve a sua coleta prejudicada por falha do aparelho de espirometria.

Sendo assim, participaram do processo de validação da EPEC um total de 36 crianças, com média de idade de $10 \pm 2,61$ anos, sendo $50 \%$ de meninas. A caracterização detalhada da amostra está descrita na TABELA 1.

O comportamento da média da pontuação da EPEC e BORG dos dois TC6 de cada dia de avaliação é apresentado na FIGURA 2. de cada dia para analisar o grau de desvio dos dados obtidos, para o qual adotou-se a seguinte fórmula: $E P M: S \sqrt{ }(1-p)$, onde $S$ : desvio padrão e p: coeficiente de Pearson. A reprodutibilidade e confiabilidade (entre avaliadores) da aplicação da EPEC foram determinadas pelo coeficiente de correlação intraclasse de duas vias (consistência) (ICC - two way mixed model, consistency). O ICC foi interpretado conforme o sistema de classificação de Munro $^{29}$, sendo pouca correlação $(<0,25)$, baixa correlação $(0,26-0,49)$, moderada $(0,50-0,69)$, alta $(0,7-0,89)$ e muito alta $(0,9$ $1,0)$. O coeficiente de Kappa foi aplicado para verificar a concordância entre as medidas de cada avaliador nos dois dias de aplicaçáo do protocolo, e a interpretaçâo dos resultados foi realizada de acordo com LANDis e $\mathrm{KoCH}^{30}$. A disposição gráfica de Bland e Altman ${ }^{31}$ complementou a análise da reprodutibilidade e confiabilidade, por permitir melhor visualizaçáo da concordância entre as medidas individuais. Para avaliação da fidelidade utilizou-se o alpha de Cronbach, no sentido de se analisar a consistência interna deste instrumento, a qual indica a relação entre os itens da escala. Segundo Ribeiro et al..$^{32}$, uma boa consistência interna deve exceder um $\alpha$ de 0,80 . O nível de significância adotado para o tratamento estatístico foi de 5\% ( $<<0,05)$.
Os resultados de validação da EPEC, no primeiro e segundo dia, de ambos avaliadores, estão descritos na TABELA 2. A TABELA 3 apresenta os dados de ICC e reprodutibilidade. Observa-se de moderada a alta correlaçáo intraclasse da EPEC entre os dois avaliadores nos dois dias, demonstrados pelos gráficos de Bland \& Altman (FIGURA 3).

No cálculo do EPM, encontrou-se um valor de 0,05 para o primeiro dia e 0,03 no segundo dia. $\mathrm{O}$ EPE nos dois dias foi de 0,33 no primeiro dia e 0,41 no segundo dia.

O coeficiente alfa de Cronbach foi de 0,865 no primeiro dia e 0,862 no segundo dia, informando que a consistência interna da EPEC é muito boa. O resultado do coeficiente de Kappa caracterizou moderada concordância entre as medidas dos dois avaliadores, sendo no primeiro dia K: 0,384 e no segundo dia K: 0,338, em ambas avaliações a concordância foi moderada. 
TABELA 1 - Caracterização da amostra segundo idade, dados biométricos e espirométricos.

\begin{tabular}{lcc}
\hline Variáveis & Média \pm dp & Mín.-Máx. \\
\hline Idade (anos) & $10,00 \pm 2,61$ & $6-14$ \\
Peso $(\mathrm{Kg})$ & $39,1 \pm 12,86$ & $22,00-65,20$ \\
Altura $(\mathrm{cm})$ & $141,26 \pm 14,14$ & $114,00-167,00$ \\
$\mathrm{IMC}(\mathrm{Kg} / \mathrm{m} 2)$ & $19,15 \pm 3,79$ & $13,10-27,03$ \\
$\mathrm{VEF}_{1} \%$ & $93,83 \pm 9,94$ & $80-118$ \\
$\mathrm{CVF}_{0}$ & $99,36 \pm 10,322$ & $84-122$ \\
$\mathrm{VEF}_{1} / \mathrm{CVF} \%$ & $86,22 \pm 5,26$ & $73-99$ \\
$\mathrm{DP}$ & $558,73 \pm 90,55$ & $422,30-750,00$ \\
\hline
\end{tabular}

dp: desvio padrão Mín.: mínimo;

Máx.: máximo;

Kg: quilogramas;

cm: centímetros;

IMC: índice de massa

corporal;

ASC: área de superfície

corporal;

m2: metros quadrado;

MID: membro inferior

direito;

MIE: membro inferior

esquerdo;

VEF: volume expiratório

forçado no primeiro

segundo;

CVF: capacidade vital

forçada;

$\%$ : porcentagem do

predito;

DP: distância percorrida

no melhor teste de

caminhada de seis

minutos.
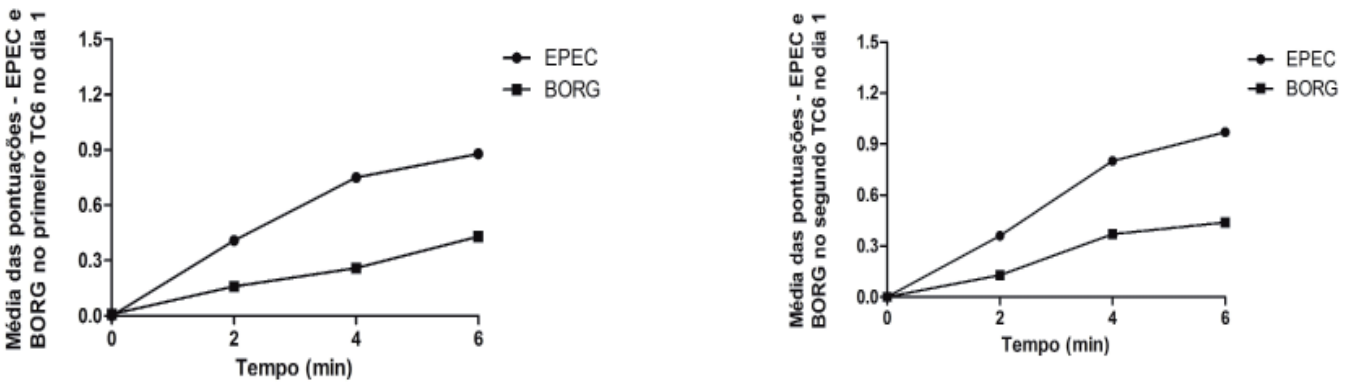

EPEC: Escala de percepção de esforço para crianças; BORG: Escala modificada de BORG; TC6: Teste de caminhada de seis
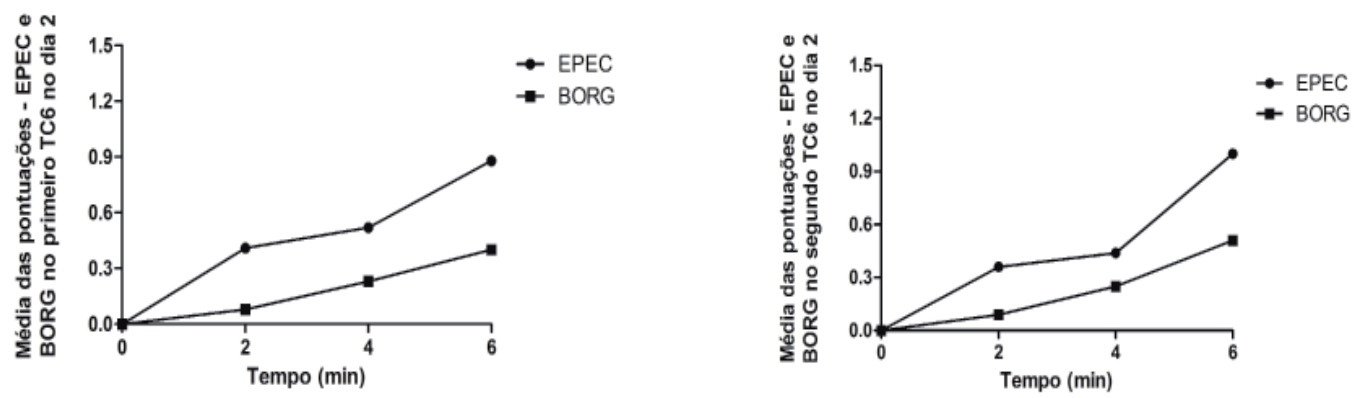

FIGURA 2 - Comportamento da pontuação das duas escalas de esforço apontada pelas crianças nos 4 testes de caminhada de seis minutos. 
Martins R, et al.

A1 - avaliador 1;

A2 - avaliador 2;

D1 - dia 1;

D2 - dia2;

i-medida inicial;

2 - medida no segundo

minuto;

4 - medida no quarto

minuto;

$\mathrm{f}$ - medida final;

$r$ - índice de correlação;

p- significância estatística.

TABELA 2 - Resultado do coeficiente de correlação de Pearson entre os dados da EPEC e da escala BORG, nos dois TC6, e na análise feita pelos 2 avaliadores.

\begin{tabular}{lcc}
\hline & $\mathbf{r}$ & $\mathbf{p}$ \\
\hline EPECi x BORGi D1A1 & 1,000 & 0,000 \\
EPEC2x BORG2 D1A1 & 0,683 & 0,000 \\
EPEC4 x BORG4 D1A1 & 0,584 & 0,000 \\
EPECf x BORGf D1A1 & 0,686 & 0,000 \\
EPECi x BORGi D1A2 & 1,000 & 0,000 \\
EPEC2x BORG2 D1A2 & 0,525 & 0,001 \\
EPEC4 x BORG4 D1A2 & 0,786 & 0,000 \\
EPECf x BORGf D1A2 & 0,726 & 0,000 \\
EPECi x BORGi D2A1 & 1,000 & 0,000 \\
EPEC2x BORG2 D2A1 & 0,590 & 0,000 \\
EPEC4 x BORG4 D2A1 & 0,873 & 0,000 \\
EPECf x BORGf D2A1 & 0,761 & 0,000 \\
EPECi x BORGi D2A2 & 1,000 & 0,000 \\
EPEC2x BORG2 D2A2 & 0,653 & 0,000 \\
EPEC4 x BORG4 D2A2 & 0,892 & 0,000 \\
EPECf x BORGf D2A2 & 0,803 & 0,000
\end{tabular}

ICC: coeficiente de

TABELA 3 - Resultado do coeficientedecorrelação intraclasse(ICC) na aplicação da EPECfeita pelos 2 avaliadores. correlação intraclasse; IC: intervalo de confiança;

LI: limite inferior;

LS: limite superior;

p: valor de "p";

A1 - avaliador 1 ;

A2 - avaliador 2;

D1 - dia 1;

\begin{tabular}{cccc}
\hline & ICC & IC (lim. sup. - lim. inf. & p \\
\hline A1 x A2 D1 & 0,598 & $0,340-0,773$ & 0,000 \\
A1 x A2 D2 & 0,804 & $0,649-0,896$ & 0,000 \\
\hline
\end{tabular}

D2 - dia2 
A
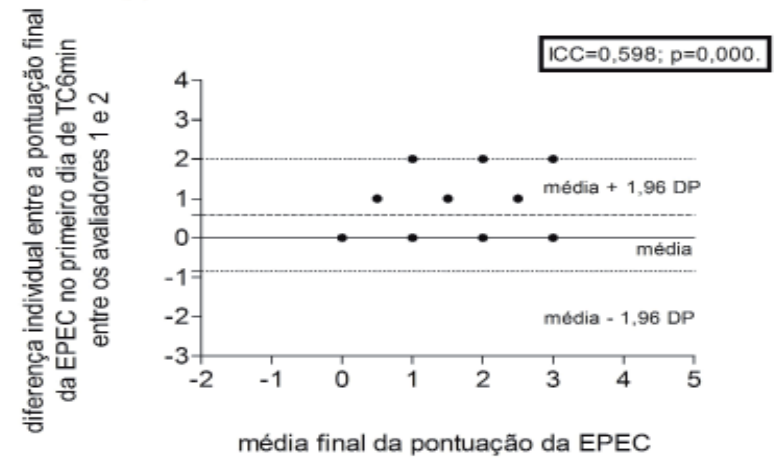

Distribuição das diferenças individuais entre a pontuação final da Escala de Percepção de Esforço para Crianças (EPEC) no teste de caminhada de seis minutos (TC6min) e as médias individuais da pontuação

B
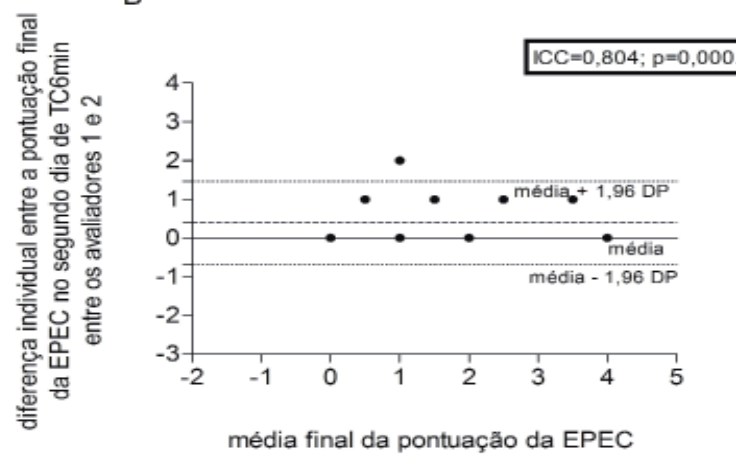

final da EPEC entre

os avaliadores 1 e 2

em ambos os dias de

avaliação (A e B).

(A) Média das diferenças

$=0,583 /$ Desvio padrão

(DP) $=0,731($ IC95\%

$-0,849-2,015)$. (B) Média

das diferenças $=0,388$

I DP = 0,549 (IC95\%

$-0,688-1,464)$.

FIGURA 3 - Correlação intraclasse (ICC) da EPEC entre os dois avaliadores nos dois dias.

\section{Discussão}

Para a população pediátrica, a grande maioria dos instrumentos de pesquisa dependem do entendimento da criança para uma mensuração fidedigna. Em se tratando da avaliação da sensação de dispneia ou de esforço, isso não é diferente. No entanto, no cenário brasileiro, apenas uma escala desse gênero foi traduzida ${ }^{33}$ até o momento, e nenhuma validada, motivo pelo qual se justifica a presente pesquisa com a PES-C, e consequente EPEC.

A PES-C foi desenvolvida para avaliar a percepção de esforço em crianças e validada com crianças saudáveis de 6 e $7 \operatorname{anos}^{18}$. No presente estudo, validou-se essa escala em crianças brasileiras, de 6 a 14 anos, incluindo-se um maior intervalo de idade, uma vez que vários estudos relacionados a testes de exercícios submáximos são conduzidos nessa faixa etária, carecendo de instrumentos de controle $28,35,37$.

Para a realização da validação da EPEC, utilizou-se a escala modificada de BORG, por ser utilizada na maioria dos estudos que envolvem a avaliação da percepção do esforço ${ }^{3,4,6,35-37}$, bem como por avaliar o mesmo constructo, sensação de dispneia e esforço ${ }^{38}$. Após a análise de dados, verificou-se a confiabilidade da média amostral por meio do EPM, a qual apresentou um valor de 0,05 para o primeiro dia e 0,03 no segundo dia, demonstrando pequena variabilidade. $\mathrm{O}$ controle do baixo erro na aplicação da escala foi caracterizado pelo EPE de 0,33 no primeiro dia e 0,41 no segundo dia, e coeficiente de Kappa constatou moderada concordância entre as medidas dos dois avaliadores. Esses achados 
parecem constatar sua validação para língua portuguesa do Brasil durante o TC6.

Durante o processo de validação da escala, identificou-se dificuldades por parte da amostra em compreender a escala modificada de BORG. As mesmas dificuldades foram encontradas na pesquisa de Hommerding e colaboradores ${ }^{10}$. Os autores realizaram um estudo com o objetivo avaliar a acurácia da escala modificada de Borg para estimar o comprometimento pulmonar de crianças e adolescentes com fibrose cística. Ao selecionar indivíduos de 6 a 10 anos e avaliar a percepção subjetiva de dispneia antes e depois de exercício submáximo, os dados foram correlacionados com a função pulmonar e TC6. Os autores constataram fraca correlaçáo da escala de Borg com as outras variáveis, principalmente em crianças menores de 9 anos.

Nessa mesma linha, outros estudos realizados com crianças que utilizam escalas como a de Borg ou sua modificação, não apresentam relaçôes significativas entre a percepção de esforço e o exercício que está sendo proposto ${ }^{2,6,10,34,36}$. Diante da dificuldade de aplicação desses instrumentos para as crianças, os quais são caracterizados por palavras, LANG-Chen et al. ${ }^{35}$ defendem que essa população compreende melhor expressóes faciais. A PESC-C e EPEC apresentam seis ilustraçóes descritivas, posicionadas ao longo da escala na forma de um urso, além dos descritores verbais, o que lhe confere maior afinidade para uso em pediatria. A postura e a expressáo facial da ilustração do urso vão se alterando à medida que a intensidade do esforço aumenta ${ }^{18}$.

Corroborando com essa ideia, a corrente investigação identificou uma maior alteração da EPEC, em comparação a escala de Borg, à medida que a criança progrediu no TC6. Este achado sugere maior facilidade das crianças em identificar o esforço frente ao instrumento ilustrado e aponta para necessidade de outras pesquisas, incluindo testes de esforço mais vigoroso, como o shuttle walk test (SWT), para ampliar a análise da sensação de esforço em testes máximos ${ }^{37}$.

Esse tipo de estudo, aplicação do EPEC durante o SWT, pode ser complementado com uma análise concomitante do comportamento dos parâmetros fisiológicos, a qual não foi feita na corrente pesquisa e pode ser considerada uma limitação, pois o processo de validação restringiuse apenas a comparaçáo com um instrumento que avaliasse o mesmo constructo que a PES-C. Outra limitaçáo deve-se a ampla faixa etária da amostra, sem controle do processo de maturação sexual, uma vez que a inclusão de adolescentes pode ter influenciado na compreensão e nos resultados obtidos. O ICC moderado inter avaliadores no primeiro dia, ainda que a validação da EPEC tenha apresentado reprodutibilidade, consistência interna, baixas variabilidade e erro em sua aplicação, também deve ser considerado.

Sumarizando, a presente pesquisa apresentou o resultado da tradução da PES-C para o português do Brasil e validação da EPEC para a população de crianças brasileiras durante o TC6 e atestou, por meio da análise estatística, que se trata de um instrumento reprodutível, consistente e de aplicação segura nessa população, o que pode caracterizá-la como uma alternativa confiável na avaliação da percepção de esforço em pediatria. 


\section{Abstract}

Child perception perception scale (EPEC): validation for Portuguese in a submaximal test

The aim of study was translate and validate, paragraph Portuguese of Brazil, a scale Perception effort to Children. The translation was based on stage of PES-C, the English language to a Portuguese (Brazil), was based in any protocol proposed by Guillemin et al. ${ }^{19}$. To scale validation, PES-C was applied during Walk Test procedure of SIX Minutes (6MWT). The validity of EPEC was evaluated using the Pearson correlation coefficient to verify the degree of association with the Borg scale. For the stability of the responses, the standard error of the mean (SEM) and ICC (intra-class correlation coefficient) were calculated. The standard error of the estimation of the final perception measurements (EEP) of each day was calculated to analyze the degree of deviation of the obtained data. For fidelity assessment was used Cronbach's alpha, not SENSE to analyze an internal Consistency. In this article, validation Process 36 children participated in the PES-C, with average age of $10 \pm 2.61$ years, being 50\% Girls. In the EPM calculation, a value of 0.05 was found for the first day and 0.03 for the second day, characterizing a low variability. The SEM was 0.33 on the first day and 0.41 on the second day, demonstrating a low error. Internal consistency, Cronbach's alpha coefficient was 0.865.The scale was validated paragraph one EPEC Children Brazilian population, demonstrating also be reproducible.

KEYwords: Reproducibility; Young, Stress; Translation.

\section{Referências}

1. Borg GAV. Psychophysical bases of perceived exertion. Med Sci Sports Exerc. 1982;14(5):377-81.

2. American College of Sports Medicine. Diretrizes do ACSM para os testes de esforço e sua prescrição. 3a. ed. Rio de Janeiro: Guanabara Koogan; 2003.

3. Rodríguez-NúnezIván, Manterola Carlos. Validación inicial de la escala de medición de esfuerzopercibido infantil (EPInfant) enniños chilenos. Biomédica. 2016; 36(1):29-38.

4. Nakamura FY, Gancedo MR, Silva LA, Lima JRP, Kokubun E. Utilização do esforço percebido na determinação da velocidade crítica em corrida aquática. Rev Bras Med Esporte. 2005;11(1):1-5.

5. Borg GAV, Noble BJ. Perceived exertion. Wilmore JH, editor. Exercise and sport sciences reviews. Acad Press. 1974;2(1):131-53.

6. Martins R, Assumpção MS, Schivinski CIS. Percepção de esforço e dispneia em pediatria: revisão das escalas e avaliação. Medicina (RibeirãoPreto). 2014;47(1):25-35.

7. Borg G. A category scale with ratio properties for intermodal and interindividual comparisons. In: Proceedings of the 22nd International Congress of Psychology; 1980. Berlin: VEB DeutscherVerlag der Wissenschaften. p. 25-34.

8. Granja JC, Paschoal MA. Relação entre o índice de percepção de esforço de Borg, nível 13 (ligeiramente cansativo), e a intensidade de esforço realizado por crianças eutróficas e obesas mórbidas. In: Anais do XV Encontro de Iniciação Científica da PUC-Campinas; 2010.

9. Oliveira JSD, Campos TF, Borja RDO, Silva ROED, Freitas DAD, Oliveira LCD, Mendonça KMPPD. Análise do índice de percepção de esforço na avaliação das pressôes respiratórias máximas em crianças e adolescentes. Rev Bras Cresc Desenvolv Hum. 2012;22(3):314-20.

10. O'Sulivan SB. Perceived Exertion: a review. Am Phys Ther. 1984;64(3):343-6.

11. Hommerding PX, Donadio MV, Paim TF, Marostica PJ. The Borg scale is accurate in children and adolescents older than 9 years with cystic fibrosis. Resp Care. 2010;55(6):729-33.

12. Rogers D, Prasad SA, Doull I. Exercise testing in children with cystic fibrosis. J R Soc Med. 2003;96(43):23-9.

13. Oliveira KMC, Macêdo TMF, Borja RO, Nascimento RA, Medeiros Filho WC, Campos TF et al. Força muscular respiratória e mobilidade torácica em crianças e adolescentes com leucemia aguda e escolares saudáveis. Rev Bras Cancerol. 2011;57(4):511-7.

14. Lima PB, Santoro IL, Caetano LB, Cabral ALB, Fernandes ALG. Desempenho de uma escala analógica visual legendada na determinaçáo do grau de dispneia durante teste de broncoespasmo induzido por exercício em crianças e adolescentes 
asmáticos. J Bras Pneumol. 2010;36(5):532-538.

15. Williams JG, Eston R, Furlong B. CERT: A perceived exertion scale for young children. Percept Mot Skills. 1994;79(3f):1451-58.

16. Yelling M, Lamb KL, Swaine IL. Validity of a pictorial perceived exertion scale for effort estimation and effort production during stepping exercise in adolescent children. Eur Phys Educ Rev. 2002;8(2):157-75.

17. Robertson RJ, Goss FL, Boer NF, Peoples JA, Foreman AJ, Dabayebeh IM, Millich NB, Balasekaran G, Riechman SE, Gallagher JD, Thompkins T. Children's OMNI scale of perceived exertion: mixed gender and race validation. Med Sci Sports Exerc. 2000;32(10):452-58.

18. Simon S, Alison J, Dwyer G, Follett J. Validation of a perceived exertion scale for young children. In: Australian Physiotherapy Association-National Paediatric Conference; 2003; Perth, WA.

19. Guillemin F, Bombardier C, Beaton D. Cross-cultural adaptation of health-related quality of life measures: literature review and proposed guidelines. J Clin Epidemiol. 1993;46(12):1417-32.

20. American Thoracic Society. ATS statement: guidelines for the six-minute walk test. Am J Respir Crit Care Med. 2002;166(1):111-17.

21. Rocha ES, Rosa GJ, Schivinski CIS. Nível de atividade física e funcional de crianças atletas. Rev Bras Cresc Desenvol Hum. 2014; 24(2):127-134.

22. Solé D, Vanna AT, Yamada E, Rizzo MCV, Naspitz CK. International study of asthma and allergies in childhood (ISAAC) written questionnaire: validation of the asthma component among Brazilian children. J Investig Allergol Clin Immunol. 1998;8(6):376-82.

23. Knudson RJ, Slatin RC, Lebowitz MD, Burrows B. The maximal expiratory flow-volume curves. Normal standards variability and effect of age. Am Rev Respir Dis. 1976;113(6):587-600.

24. Polgar GJ, Weng TR. The functional development of the respiratory system. Am Rev Respir Dis. 1979;120(3):625-95.

25. Puccini RF, Bresolin AMB. Dores recorrentes na infância e na adolescência. J Pediatr. 2003;79(1):S65-S76.

26. Miller MR, Hankinson J, Brusasco V, Burgos F, Casaburi R, Coates R, et al. ATS/ERS: Standardisation of spirometry. Europ Respir Soc. 2005;26(2):319-38.

27. Cavalcante TMC, Diccini S, Barbosa DA, Bittencourt ARC. Uso da escala modificada de Borg na crise asmática. Acta Paul Enferm. 2008;21(3):466-73.

28. Li AM, Yin J, Yu CCW, Tsang T, HK SO, Wong E, Chan D, Hon EKL, Sung R. The six-minute walk test in healthy children: reliability and validity. Eur Respir Soc. 2005;25(6):1057-1060.

29. Munro, B.H. Statistical methods for health care research. 3a. ed. New York: Lippincott Williams \&Wilkins; 1997.

30. Landis JR, Koch GG. The measurement of observer agreement for categorical ata. Biometrics.1977;33:159-174.

31. Bland JM, Altman DG. Statistical methods for assessing agreement between two methods of clinical measurement. Lancet. 1986;327(8476):307-10.

32. Ribeiro JPL. Investigação e avaliação em psicologia e saúde. 1a. ed. Lisboa: Climepsi Editores; 1999.

33. Costa GM, Dantas EHM, Marques MB, Novaes JF. Percepçáo subjetivo do esforço. Classificaçáo do esforço percebido: proposta de utilização da escala de faces. Fit Perf J. 2004; 3(6):306-309

34. Watanabe FT, Koch VHK, Juliani RCP, Cunha MT. Six-minute walk test in children and adolescents with renal diseases: tolerance, reproducibility and comparison with healthy subjects. Clinics. 2016;71(1):22-27.

35. Lang Chen Y, Chiou WK, Tzenq YT, Lu CY, Chen SC. A rating of perceived exertion scale using facial expressions for conveying exercise intensity for children and young adults. J Sci Med Sport. 2017;20(1):66-9.

36. Hostyn SV, Werther BC, Johnston C, Braga JAP. Evaluation of functional capacity for exercise in children and adolescents with sickle cell disease through the Six Minute Walk Test. J Pediatr. 2013;89(6):588-594.

37. Gonçalves CG, Mesquita R, Hayashi D, Merli MF, Vidotto LS, Fernandes KB, Probst VS.Does the incremental shuttle walking test require maximal effort in healthy subjects of different ages? Physiother. 2015;101(2):141-6.

38. Pasquali L. Psychometrics. Rev Esc Enferm USP. 2009;43:992-999.

\begin{tabular}{r|l} 
ENDEREÇo & \\
Renata Maba Gonçalves & Submetido: 11/05/2017 \\
Rua Paschoal Simone, 358 - Coqueiros & Revisado: 28/ 12/2017 \\
88080-350 - Florianópolis - SC - Brasil & Aceito: 22/08/2018 \\
E-mail: renata.maba@gmail.com &
\end{tabular}

